\title{
Matrix Differentiation of the Characteristic Function
}

\author{
By H. W. Turnbull, St Andrews University.
}

\section{(Received 12th January 1931. Read 16th January 1931.)}

The following work is a sequel to three previous communications, ${ }^{1}$ and more particularly to the first. The present object is to shew the effect of repeated operation with the matrix differential operator $\Omega \equiv\left[\frac{\partial}{\partial x_{j i}}\right]$, when it acts upon a scalar matrix formed from an $n$ rowed determinant $\left|x_{i j}\right|$, or sums of principal minors, the $n^{2}$ elements $x_{i j}$ being treated as independent variables. Thus when $z$ is a scalar quantity $\Omega z$ means the matrix $\left[\partial z / \partial x_{j i}\right]$, whose $i j^{\text {th }}$ element is the derivative $z / \partial x_{j i}$.

\section{§1. Fundamental Formulae.}

From the square matrix

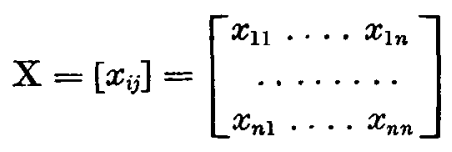

there may be derived a determinant $|X|$ and a characteristic function $\phi(\lambda)$, given by

$$
\begin{aligned}
\phi(\lambda) & \equiv|\lambda U-X| \equiv\left|\begin{array}{c}
\lambda-x_{11} \ldots \ldots-x_{1 n} \\
\ldots \ldots \ldots \ldots \ldots \\
-x_{n 1} \ldots \ldots \lambda-x_{n n}
\end{array}\right| \\
& =p_{0} \lambda^{n}+p_{1} \lambda^{n-1}+\ldots \ldots+p_{n-1} \lambda+p_{n} .
\end{aligned}
$$

Clearly $p_{n}$ is equal to $(-)^{n}|X|$, while $p_{0}=1$. The reciprocal of this polynomial $\phi(\lambda)$ can be expanded in the form

$$
\psi(\lambda)=\frac{1}{\phi(\lambda)}=\frac{h_{0}}{\lambda^{n}}+\frac{h_{1}}{\lambda^{n+1}}+\frac{h_{2}}{\lambda^{n+2}}+\ldots
$$

I I. H. W. Turnbull, On differentiating a matrix, Proc. Edinburgh Math. Soc. (2), 1 (1927), 111-128.

II. A matrix form of 'Taylor's Theorem (2), 2 (1929), 33-54.

III. The invariant theory of bilinear forms, Proc. London Math. Soc. (1931). 
for suitably large values of the modulus of $\lambda$, where the coefficients $h_{r}$ are homogeneous products of the $n$ latent roots $\lambda_{i}$ of $X$, defined by $\phi\left(\lambda_{i}\right)=0$. The coefficients $p$ and $h$ satisfy the well known Wronskian relations

$$
h_{r} p_{0}+h_{r-1} p_{1}+h_{r-2} p_{2}+\ldots+h_{1} p_{r-1}+h_{0} p_{r}=0,
$$

where $r=1,2, \ldots$ The unit matrix is denoted by $I=\left[\delta_{i j}\right]$ in terms of the Kronecker delta; and an arbitrary constant matrix by $A=\left[a_{i j}\right]$. Bith $\lambda$ and the $a_{i j}$ are independent of the $x_{i j}$, whereas the $h_{r}$ and $p_{r}$ are clearly functions of the $x_{i j}$. As usual $s_{r}$ denotes the sum of the $r^{\text {th }}$ powers of the $n$ latent roots $\lambda_{i}$.

By $\Omega \theta$ is meant the matrix $\left[\partial \theta / \partial x_{j i}\right]$ whose $i j^{\text {th }}$ element is $\partial \theta / \partial x_{j i}$, $\theta$ being a scalar quantity. Taking $\theta$ to be $s, p$ and $h$ in turn, the fundamental formulae of $\Omega$ differentiation ( $C f . I, p .119)$ are

$$
\begin{gathered}
\Omega s_{r}=r X^{r-1}, \\
P_{r}=X^{r}+p_{1} X^{r-1}+\ldots+p_{r-1} X+p_{r} I=-\Omega p_{r+1}, \\
H_{r}=X^{r}+h_{1} X^{r-1}+\ldots+h_{r-1} X+h_{r} I=\Omega h_{r+1} .
\end{gathered}
$$

It is useful to have a special notation $P$ and $H$ for these polynomial scalar functions of the matrix $X$, whose order is shewn by the suffix. Initially $r$ is taken to be zero or a positive integer, so that $P_{0}=H_{0}=I$; when $r \geqslant n$, the right member of (7) disappears, $p_{r}$ being zero, and the Cayley Hamilton equation

$$
P_{n} \equiv \phi(X) \equiv X^{n}+p_{1} X^{n-1}+\ldots+P_{n-1} X+P_{n} I=0
$$

is put in evidence.

The reciprocal properties (7) and (8) are brought out very clearly by the following new proof, which is based on the inverse of the $\lambda$-matrix $\lambda I-X$.

Letting $X_{i j}$ denote the cofactor of $x_{i j}$ in the determinant $|X|$, we may write the reciprocal of the non-singular matrix $X$ in the form

But we have

$$
X^{-1}=\left[X_{j i} \mathrm{j} /|X|\right. \text {. }
$$

$$
X_{j i}=\frac{\partial}{\partial x_{j i}}|X|
$$

hence

$$
\left[X_{j i}\right]=\Omega|X|, \quad X^{-1}=\Omega|X| /|X| .
$$


Let each $x_{i j}$ be replaced by $x_{i j}-\lambda a_{i j}$, where $\lambda$ and $a_{i j}$ are constants. This leaves $\partial / \partial x_{j i}$, and therefore $\Omega$ unaltered, but replaces the matrix $X$ by $X-\lambda A$. Accordingly we have the relation

$$
\frac{1}{\bar{X}-\lambda A}=\frac{\Omega|X-\lambda A|}{|\bar{X}-\lambda A|}
$$

identically for all values of $\lambda$ and $a$, a result which can also be exhibited as

$$
\frac{1}{X-\lambda A}=\Omega \log |X-\lambda A| \text {. }
$$

In particular let $A$ be replaced by the unit matrix $I$. Then

$$
\begin{aligned}
-\log |X-\lambda \boldsymbol{I}| & =-\log \left(\lambda_{1}-\lambda\right)\left(\lambda_{2}-\lambda\right) \ldots\left(\lambda_{n}-\lambda\right) \\
& =-\log (-\lambda)^{n}+\frac{s_{1}}{\lambda}+\frac{s_{2}}{2 \lambda^{2}}+\frac{s_{3}}{3 \lambda^{3}}+\ldots
\end{aligned}
$$

for large enough values of the modulus of $\lambda$, while

$$
-(X-\lambda \boldsymbol{I})^{-1}=\frac{I}{\lambda}+\frac{X}{\lambda^{2}}+\frac{X}{\lambda^{3}}+\cdots
$$

Result (6) follows at once by substituting these values in (14) and comparing coefficients of corresponding negative powers of $\lambda$. More generally, if $A^{-1}=C$, the same procedure leads to the relation

$$
\Omega_{s}(C X)^{r}=r(C X)^{r-1} C
$$

in the notation of II, p. 37 .

To obtain the relation (7), let (13) be written in the form

$$
\frac{|\lambda A-X|}{\lambda A-X}=-\Omega|\lambda A-X| \text {. }
$$

Treating numerator and denominator of the left member as a polynomial and a linear function of $\lambda$, we may perform ordinary long division in every case when $A$ commutes with $X$. This is so when $A=I$, making the left member $\phi(\lambda) \div(I \lambda-X)$. The polynomial $\phi(\lambda)$ is given by (3); on carrying out the long division the result is

$$
\begin{aligned}
\frac{\phi(\lambda)}{I \lambda-X}=I \lambda^{n-1}+\left(X+p_{1} I\right) & \lambda^{n-2}+\ldots+\left(X^{n-1}+\ldots+p_{n-1} I\right) \\
& +\frac{\phi(X)}{I \lambda-X} \\
= & P_{0} \lambda^{n-1}+P_{1} \lambda^{n-2}+\ldots+P_{n-1}+\frac{P_{n}}{I \lambda-X} .
\end{aligned}
$$


Again from the right member of (16), with $A=I$, we obtain

$$
-\left(\lambda^{n-1} \Omega p_{1}+\lambda^{n-2} \Omega p_{2}+\ldots+\Omega p_{n}\right) \text {, }
$$

since $\Omega \lambda^{n}=0$. On multiplying throughout, here and in (17), by $I \lambda-X$, expanding, and equating coefficients of powers of $\lambda$, we obtain the relations (7), and also the Cayley Hamilton theorem implied by $\phi(X)=0$.

Reciprocally, since $\phi(\lambda) \psi(\lambda)=1$, it follows that

but, since

$$
\{\Omega \phi(\lambda)\} \psi(\lambda)+\phi(\lambda) \Omega \psi(\lambda)=0 ;
$$

we have

$$
\phi(\lambda)=|\lambda I-X|,
$$

$$
\frac{\Omega \phi(\lambda)}{\phi(\lambda)}=\frac{1}{X-\lambda I}=-\frac{\Omega \psi(\lambda)}{\psi(\lambda)} .
$$

Again by ordinary long division of the series (4) by $I-X \lambda^{-1}$, arranged in descending powers (all negative) of $\lambda$, we have

$$
\begin{aligned}
& \frac{\psi(\lambda)}{I-X \lambda^{-1}}=\lambda^{-n}+\left(X+h_{1} I\right) \lambda^{-n-1}+\left(X^{2}+h_{1} X+h_{2} I\right) \lambda^{-n-2}+\ldots \\
& \text { Also } \\
& =H_{0} \lambda^{-n}+H_{1} \lambda^{-n-1}+H_{2} \lambda^{-n-2}+\ldots \\
& \Omega \psi(\lambda)=h_{1} \Omega \lambda^{-n-1}+h_{2} \Omega \lambda^{-n-2}+\ldots
\end{aligned}
$$

On substituting in (18), clearing of fractions, and comparing coefficients as before, the relations (8) follow. Incidentally we have the result ${ }^{1}$

$$
\begin{aligned}
\frac{1}{I \lambda-X} & =\frac{P_{0} \lambda^{n-1}+P_{1} \lambda^{n-2}+\ldots .+P_{n-2} \lambda+P_{n-1}}{\left(\lambda-\lambda_{1}\right)\left(\lambda-\lambda_{2}\right) \ldots\left(\lambda-\lambda_{n}\right)} \\
& =\left(H_{0}+H_{1} \lambda^{-1}+H_{2} \lambda^{-2}+\ldots\right)\left(\lambda-\lambda_{1}\right) \ldots\left(\lambda-\lambda_{n}\right) \lambda^{-n-1} .
\end{aligned}
$$

These coefficients $P$ and $H$ are matrices which commute with $X$ and with each other, since they are polynomials in $X$. From this relation each $P_{r}$ can be deduced as a linear function of the $H_{x}$ with $s \leqslant r$, the coefficients being polynomial expressions in the $p$ 's. Correlatively for $H$ in terms of $P$. Also if the $r^{\text {th }}$ Wronskian relation (5) is written $w_{r}(h, p)=0$, it follows that

$$
w_{r}(H, p)=w_{r}(h, P) .
$$

For example $H_{2} p_{0}+H_{1} p_{1}+H_{0} p_{2}=h_{2} P_{0}+h_{1} P_{1}+h_{0} P_{2}$.

${ }^{1}$ Of. L. E. Dickson, Modern Algebraic Theories (Chicago, 1926), 48, after replacing $P_{r}$ by $C_{n-1-r}$. 


\section{§2. The Converse Problem.}

By solving the recurrence relations (7) and (8) for successive powers of $X$ we obtain the following equations, in which an accent denotes the effect of the $\Omega$ operation:

$$
\begin{aligned}
-p_{1}{ }^{\prime} & =I=h_{1}{ }^{\prime}, \\
-h_{1} p_{1}{ }^{\prime}-p_{2}{ }^{\prime} & =X=h_{2}{ }^{\prime}+h_{1}{ }^{\prime} p_{1}, \\
-h_{2} p_{1}{ }^{\prime}-h_{1} p_{2}{ }^{\prime}-p_{3}{ }^{\prime} & =X^{2}=h_{3}{ }^{\prime}+h_{2}{ }^{\prime} p_{1}+h_{1}{ }^{\prime} p_{2},
\end{aligned}
$$

and in general (since $p_{0}{ }^{\prime}=h_{0}{ }^{\prime}=0$ ),

$$
-w_{r}\left(h, \dot{p}^{\prime}\right)=X^{r-1}=w_{r}\left(h^{\prime}, p\right)
$$

These follow at once from (18), on multiplying throughout by $\phi(\lambda) \psi(\lambda)$ (which is unity), then expanding each of the three expressions in descending powers of $\lambda$, and again equating coefficients. These alternative expressions for a power of $X$ lead to the theorem:

The $(r-1)^{\mathrm{th}}$ power of a matrix $X$ is obtained by $\Omega$ differentiation from the $r^{\text {h }}$ Wronksian relation, either by treating the $p$ 's as constants, or else by treating the $h$ 's as constants and affixing a negatice sign to the result.

\section{§3. Successive $\Omega$ differentiation.}

THEOREM I. Any two consecutive coefficients $p_{r}, p_{r+1}$ of the characteristic function $\phi(\lambda)$ satisfy the matrix differential equation

$$
\Omega^{2} p_{r+1}=(n-r) \Omega p_{r}
$$

Proof. The left member of this equation denotes the effect of $\Omega$ operating upon $\Omega p_{r+1}$, and is therefore equal to

$$
-\Omega\left(X^{r}+p_{1} X^{r-1}+\ldots+p_{r-1} X+p_{r} I\right) .
$$

Now, by I, p. 117 (2),

$$
\Omega X^{v}=s_{0} X^{v-1}+s_{1} X^{\nu-2}+\ldots+s_{\nu-1} I,
$$

where $s_{0}=n$. Also $\Omega p_{r_{-\nu}} X^{\nu}=p_{r-\nu} \Omega X^{\nu}+\left(\Omega p_{r_{-\nu}}\right) X^{\nu}$. Let this last be simplified, by use of (7) and (23), and arranged in descending powers of $X$. On summing the results for $\nu:=0,1,2, \ldots, r$ we have

where

$$
\Omega^{2} p_{r+1}=q_{0} X^{r-1}+q_{1} X^{r-2}+\ldots+q_{r-2} X+q_{r-1} I,
$$

$$
q_{m}=-\left(s_{m}+p_{1} s_{m-1}+\ldots+p_{m} s_{0}\right)+(r-m) p_{m}
$$


After using the Newtonian relation

$$
s_{m}+p_{1} s_{m-1}+\ldots+p_{m-1} s_{1}+m p_{m}=0
$$

$q_{m}$ becomes $(r-n) p_{m}$. Hence

$$
\Omega^{2} p_{r+1}=-(n-r)\left(X^{r-1}+p_{1} X^{r-2}+\ldots+p_{r-1} I\right)=(n-r) \Omega p_{r},
$$

which proves the theorem.

Theorem II. Correlatively, consecutive coefficients $h_{r+1}, h_{r}$ satisfy the equation

$$
\Omega^{2} h_{r+1}=(n+r) \Omega h_{r}
$$

Proof. The proof is analogous to that of Theorem I, but utilizes the relation

$$
s_{m}+h_{1} s_{m-1}+\ldots+h_{m-1} s_{1}=m h_{m} .
$$

As a consequence of these two theorems we may express each matrix $P_{\nu}$ and $H_{\nu}$ as a matrix derivative of $p_{\mu}$ and $h_{\mu}$, respectively, provided that the suffix $\mu$ exceeds $\nu$. For example,

$$
\Omega^{3} p_{r+1}=(n-r) \Omega^{2} p_{r}=(n-r)(n-r-1) \Omega p_{r-1} .
$$

This leads straightforwardly to the relations

$$
\begin{aligned}
\Omega^{n-r} p_{n} & =\Omega^{n-r-1} p_{n-1}=2 ! \Omega^{n-r-2} p_{n-2}=\ldots \\
& =(n-r-1) ! \Omega p_{+}=-(n-r-1) ! P_{r},
\end{aligned}
$$

where $r=0,1, \ldots, n-1$. In particul. $\mathrm{r}$, when $r=0$, the result may be written

$$
\Omega^{m} p_{m}=-(n-1) ! /(n-m) !, \quad 0<m \leqslant n,
$$

so that the effect of $m$ operations with $\Omega$ upon the coefficient $p_{m}$ in the characteristic function, yields a negative integer.

Similarly from Theorem II,

$$
H_{r}=\Omega h_{r+1}=\frac{(n+r) !}{(n+r+1) !} \Omega^{2} h_{r+2}=\frac{(n+r) !}{(n+r+2) !} \Omega^{3} h_{r+3}=\ldots
$$

Theorem III. Any power series

$$
f(X)=a_{0} I+a_{1} X+a_{2} X^{2}+\ldots
$$

with scalar coefficients $a_{i}$ can be derived from the scalar matrix $|X| I$ by means of a matrix operator $g(\Omega)$ which is a scalar polynomial, of order $n$, or less, in $\Omega$. 
Proof. On substituting for powers of $X$ from (21) we have

where

$$
f(X)=\beta_{0} p_{1}{ }^{\prime}+\beta_{1} p_{2}{ }^{\prime}+\ldots+\beta_{n-1} p_{n}{ }^{\prime},
$$

$$
\beta_{m}=-a_{m}-a_{m+1} h_{1}-a_{m+2} h_{2}-\ldots, \quad(m=0,1,2, \ldots) .
$$

Also by (25),

$$
\begin{aligned}
& p_{1}{ }^{\prime}=\Omega p_{1}=\frac{1}{(n-1)} \Omega^{2} p_{2}=\ldots=\frac{1}{(n-1) !} \Omega^{n} p_{n}, \\
& p_{2}{ }^{\prime}=\Omega p_{2}=\frac{1}{(n-2) !} \Omega^{n-1} p_{n}
\end{aligned}
$$

and so on. Hence we have

$$
\begin{aligned}
f(X) & =\left(\frac{\beta_{0} \Omega^{n}}{(n-1) !}+\frac{\beta_{1} \Omega^{n-1}}{(n-2) !}+\ldots+\frac{\beta_{n-2} \Omega^{2}}{1 !}+\beta_{n-1} \Omega\right) p_{n} . \\
& =(-)^{n} g(\Omega) p_{n}, \text { say. }
\end{aligned}
$$

The theorem follows since $p_{n}=(-)^{n}|X|$.

CoRollary. Any polynomial $f(X)$ of order $r$ less than $n$ can be derived from an earlier coefficient $p_{m}$ by an analogous operator $g_{m}(\Omega)$, whenever $m>r$.

A similar theorem holds for the derivation of a polynomial $f(X)$ from a coefficient $h_{m}$ of higher order. For example

$$
\begin{aligned}
X^{2} & =-\left(1+\frac{h_{1}}{n-2} \Omega+\frac{h_{2}}{(n-1)(n-2)} \Omega^{2}\right) \Omega p_{3} \\
& =\left(1+\frac{p_{1}}{n+2} \Omega+\frac{p_{2}}{(n+1)(n+2)} \Omega^{2}\right) \Omega h_{3} .
\end{aligned}
$$

Theorem IV. The operator $\Omega e^{\lambda} \Omega$ has the same effect upon $p_{n}=\phi(0)$, that $\Omega$ has upon the characteristic function $\phi(\lambda)$.

Proof. We have $\Omega e^{\lambda \Omega} p_{n}=\left(\Omega+\lambda \Omega^{2}+\frac{\lambda^{2} \Omega^{3}}{3 !}+\ldots\right) p_{n}$

$$
\begin{aligned}
& =\Omega\left(\lambda^{n}+p_{1} \lambda^{n-1}+\ldots+p_{n}\right), \text { by }(25), \\
& =\Omega \phi(\lambda),
\end{aligned}
$$

which proves the theorem.

We are not however entitled to deduce the equality of $e^{\lambda} \Omega p_{n}$ and $\phi(\lambda)$, by operating with $\Omega^{-1}$, since it by no means follows that when $\Omega Y=0, Y$ itself is zero. 


\section{§4. Connection with invariant theory.}

As has been pointed out in III (see Introduction), the $\Omega$ process is equivalent to polarization by use of a sum of symbolic operators

$$
\left(u \mid \frac{\partial}{\partial a}\right)\left(x \mid \frac{\partial}{\partial a}\right) \equiv\left(\sum_{i=1}^{n} u_{i} \frac{\partial}{\partial a_{i}}\right)\left(\sum_{i=1}^{n} x_{j} \frac{\partial}{\partial a_{j}}\right),
$$

where the matrix $\left[x_{i j}\right]$ is expressed in symbolic notation by various equivalents

$$
\left[x_{i j}\right]=\left[a_{i} a_{j}\right]=\left[\beta_{i} b_{j}\right]=\left[\gamma_{i} c_{j}\right]=\text { etc. }
$$

In fact $\Omega$ is given by

$$
u \Omega \xi=\Sigma\left(u \mid \frac{\partial}{\partial a}\right)\left(\xi \mid \frac{\partial}{\partial a}\right),
$$

where the summation runs through the equivalent symbols, one term for each pair $a, a$. The $i j^{\text {th }}$ element of $\Omega$ is given by the coefficient of $u_{i} \xi_{j}$ in this expression (30); and $u \Omega x$ denotes the bilinear differential form $\Sigma u_{i} \frac{\partial}{\partial x_{j i}} \xi_{j}$ in the usual matrix product notation. The quantities $p_{r}$ are now invariants of the bilinear form $\Sigma u_{i} x_{i j} \xi_{j}$; namely

$$
p_{1}=-(a \mid a), \quad p_{2}=\frac{1}{2 !}(a b \mid \alpha \beta), \quad p_{3}=-\frac{1}{3 !}(a b c \mid \alpha \beta \gamma), \ldots
$$

Since the effect of the right hand operation in (30) is to replace each pair $a, a$ in the operand by $u, x$, formulae (7) are now almost intuitive. For example

$$
u \Omega \xi p_{2}=\frac{1}{2 !}((u b \mid \xi \beta)+(a u \mid a \xi))=a_{a} u_{\xi}-a_{\xi} u_{a},
$$

since the symbols $a, \alpha$ are equivalent to $b, \beta$.

Translated back into the original notation this becomes

$$
u\left(\Omega p_{2}\right) \xi=-u\left(X+p_{1} I\right) \xi
$$

identically for all $u$ and $\xi$. Whence

$$
X+p_{1} I+\Omega p_{2}=0
$$

and similarly for all relations (7). 
Repeated $\Omega$ operation now appears as repeated polarization. For example

$$
u \Omega^{2} \xi=\Sigma\left(u \mid \frac{\partial}{\partial a}\right)\left(\frac{\partial}{\partial a} \mid \frac{\partial}{\partial b}\right)\left(\frac{\partial}{\partial \beta} \mid \xi\right),
$$

summed for all pairs of distinct equivalent symbols $a, a$ and $b, \beta$. When this acts, for example, upon $p_{3}$ it strikes out two symbols $a, b$ and two symbols $a, \beta$ in every possible way, replacing them by the single $u$ and $\xi$. This leads to the result

$$
\Omega^{2} p_{3}=(n-2) \Omega p_{2}=-(n-2)\left(X+p_{1} I\right),
$$

and similarly for other cases. 